\title{
A Study on the Analysis of Clothing CAD Parametric Technology based on the Theory of Human Body Characteristics
}

\author{
Tao Zhang \\ Jiangxi Institute of Fashion Technology, Nanchang, 330201, China
}

\begin{abstract}
Keywords: 3D clothing. Geometric constraint. 3D human model. The analysis of clothing CAD parametric technology
\end{abstract}

\begin{abstract}
With the continuous development of social mass material and cultural life, the clothing industry is just in a new stage of development. From this point of view, in its process, the realization of clothing CAD system and the continuous improvement and perfection of the clothing CAD function has become one of the greatest issues attracting most attention in society. In simple terms, clothing CAD is to introduce and apply CAD professional technology to clothing designing, clothing production, clothing production development and all aspects of clothing operation management. With the help of this mode, production quality and production efficiency of clothing can be realized. In the practical application, the enormous economic benefits brought about by the clothing CAD is, from a certain point of view, reflecting the special values of clothing CAD technology. Set in this situation, this paper, taking clothing CAD parametric technology as the object, focuses on the introduction of the theory of human body characteristics, starting from two aspects----the analysis of clothing parametric design based on constraints and the analysis of application of parameterized human model to clothing CAD. Centering on clothing CAD parametric technology based on the theory of human body characteristics, this paper presents detailed analysis and explanation, based on which this paper demonstrates that the introduction of the theory plays an vital role and is of great significance in the process of improving operation quality of clothing CAD parametric technology.
\end{abstract}

\section{Introduction}

In terms of the vigorous development of clothing CAD, network virtualization for fitting, 3D human animation and other new industries, the key to determine the development level and development quality lies in how to do simulative 3D reconstruction based on real human body structure. Especially under the background of constant updating and development of network concepts and network technology, online consumers often need network virtualization technology for fitting for the purchase of clothing, customized online clothes also calls for 3D human model of high reduction and simulation. Traditionally the body models for clothing designing involved in these links are often limited to body models of wood or plastic. From the perspective of human characteristics, there are extremely obvious differences between the standard models and consumers in body size. At the same time, the traditional human model is not suitable for the dynamic display of clothing in clothing designing in the new era. It is not difficult to find that the traditional body model should be improved and updated and its key direction of development is to make use of computer system to successfully construct human 3D models whose shape and size parameters are highly consistent with the basic information of real people and can represent human body based on human characteristics. Based on this background, aiming at the application of clothing CAD parametric technology, this paper will do a detailed analysis and description.

\section{The analysis of clothing parametric design based on constraints}

Based on analysis of the characteristics of the clothing, the constraint relationship existing in the clothing can be analyzed in detail, which mainly includes concurrent, symmetry and self symmetry. Based on the analysis of constraints relationship, the construction of geometric constraint graph with clothing as the object can more fully and effectively presented and expressed for the various types of 
geometric elements existing in 3D clothing. During this process, a constraint solving method based on constraint graph can be gained, which is the key of the study on the analysis of clothing CAD parametric technology based on the theory of human body characteristics. According to the analysis of 3D clothing geometric constraints relationship of the present stage, the common systematic constraint types of mechanical parameter can be divided into the following three types: (1). Geometric constraints: this constraint is mainly about the position relations between constrained geometric elements. The position relations mainly include horizontal, vertical, cutting and symmetry etc.; (2). The topological constraint: this type of constraint relations focuses on the dependency between reaction geometric elements; (3). Size constraints: this mainly refers to various distance dimensions marked by the designers, consisting of angles of two line and distance of two line etc..

Obviously, the connections between all kinds of constraint relations among mechanical parameters system and 3D clothing system are not closely related. For 3D clothing system, the key to apply 3D clothing parametric technology is to construct corresponding constraint information according to their own characteristics. The 3D clothing constraint relationship obtained at this stage mainly refers to the following types: 1). Concurrent constraint: mainly refers to the fact that the rings, the feature lines and the ring and feature line in 3D clothing system can meet at the same point, and in this process the common control point of feature lines and on loop parameters curves can form; (2). The symmetry constraint: mainly is the existence of a symmetry relationship between ring and ring, feature line and feature line. the corresponding elements of such constraint mainly refers to the control point on corresponding parameter curve; (3). Self symmetry constraints: mainly refers to the symmetry around the rings, front and back, left and right. Below (see Figure 1, figure 2) is the schematic diagram of constraint relation in jackets and skirts.

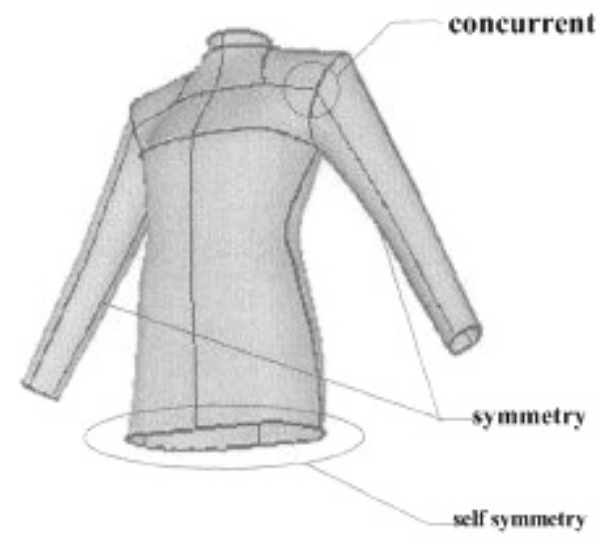

Fig. 1. Schematic diagram of three kinds of constraint relation in coats

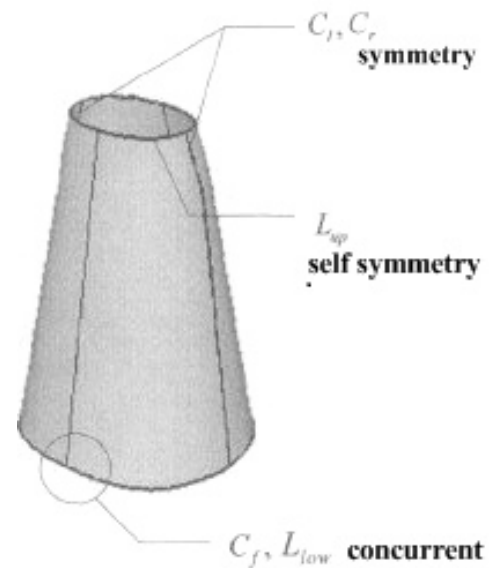

Fig. 2. Schematic diagram of three kinds of constraint relation in skirts

\section{Analysis of the application of parametric human model in clothing CAD}

Based on analysis of the general statistical structure, it is found that there is some relationship between body shape and structure in gender, height and weight were there exist. From this point of view, the division of human body can also be expressed by the differences in the height, weight and gender, and the schematic of corresponding classification are shown in the following table (see Table 1). For the most universal and representative standard 3D human body model in each classification area, the base of its accurate construction lies in obtaining the contour model closely related to the human body model, completing the extraction and interactive processing of corresponding parameter information according to the key parts of the human body model, and then establishing parametric human body model based on the theory of human characteristics. In the clothing CAD parametrization, this model can construct body surface curvature according to their own preset B spline curve, which also realizes the key objective of clothing display for any human model. 
Table 1. Division of human body model types

\begin{tabular}{|l|l|l|}
\hline gender & height $(\mathrm{mm})$ & weight $(\mathrm{kg})$ \\
\hline male/female & $>180$ & $>80$ \\
\hline male/female & $170 \sim 180$ & $70 \sim 80$ \\
\hline male/female & $160 \sim 170$ & $60 \sim 70$ \\
\hline male/female & $150 \sim 160$ & $50 \sim 60$ \\
\hline male/female & $140 \sim 150$ & $40 \sim 50$ \\
\hline male/female & $<140$ & $<40$ \\
\hline
\end{tabular}

In this process, the flow of achieving algorithm of clothing CAD parametric technology can basically be summarized as the following steps: (1). First of all, the terminal operation user should determine and input basic size information of required 3D human body model according to the needs such as gender, height, chest circumference, waist circumference, hip circumference and weight, then the terminal system will read Table 1 with the help of the basic size information and decide which category this construction of 3D human body model belongs to; (2). Secondly, the system should take the basic size information input by users in the last step as systematic parameters for common searches and comparisons in all human models in the database. In this process shaped 3D human body model consistent with the construction of 3D human body model. At the same time, the system should automatically transferred to the parameter information of basic size of shaped 3D model of human body, and then do reasonable adjustment of the positions where there are big differences between outlines of established 3D human body model and the data input by users. Specific mode for adjusting: according to the outline of shaped 3D human body model, take its center as the basic point and do linear scaling or a weight constraint processing in different directions of the basic point; (3). Again, as for the outlines that consist with those of the key parts of shaped 3D human body model, it is necessary to do reasonable scaling and adjustment according to the distance parameters preset in accordance with the outline and to the corresponding weight. The general rule in this process is: the shorter the distance is, the greater the weight generated in this process will be, and vice versa. Something more crucial is: the scaling chosen for different parts of the body in the process of adjusting is different. The realization of all the problems above needs the help of settings and restrictions of the constraint relationship; (4). Finally, after finishing adjusting the construction of human body outline, it is necessary to combine the affected and adjusted outlines, as well as the unchanged ones. Based on what have been done just now, a new set of slice information of 3D human body model outlines will be completed. And with the help of slice information, interpolation of longitudinal extension will obtain surface curvature of 3D human body model.

Based on the above analysis on algorithm of realization of clothing CAD parametric technology, it is not difficult to find that: in accordance with the information input by users, the advantages of 3D parametric human model created in dynamic mode shown in the clothing display can basically be reflected in the following respects: first of all, the 3D human body model obtained in dynamic mode comes from the most suitable basic parameters of established 3D model in systematic database of 3D features. This method ensures that the constructed human 3D will not have distortion even when in obvious dynamic adjustment, thus making system free from the possibility of malformations in the final 3D human model, and effectively improving the overall efficiency of the production of 3D human body model; secondly, in the process of implementing the algorithm of the clothing of CAD parametric technology, in the construction and adjustment of the outlines information of the differences between the construction of 3D human model and the shaped 3D model database, a constraint mechanism is used so as to ensure that each part of the 3D human body model in the whole construction process can reasonably change in accordance with the corresponding weight parameters. Obviously this method, compared with the traditional treatment of linear scaling, has a more obvious sense of reality. Below (see Figure 3) is the real effect after the application of outline interpolation for 3D model of human body surface curvature. The effect also reflects one aspect of the problem for us: during the application of the clothing CAD parametric technology explained in this paper, the outline slice obtained through combining the construction of 3D human body model is a cutting edge based 
on arbitrary spatial angle, so it can build a partial outline slice substantially vertical to skeletal structure of human during the whole construction of 3D body model. From the angle of theory of human characteristics, this approach can make use of animation to process human inherent physical changes in a simpler and more real way, eventually obtaining the animation of soft body presented in a more concrete and real way.

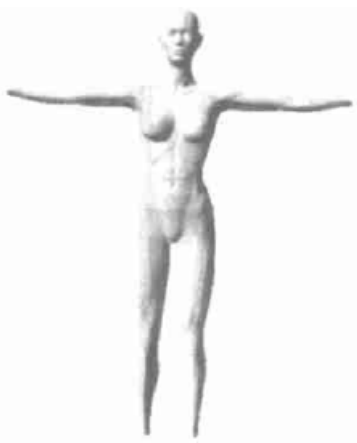

Fig. 3. Diagram of the effect of application of the outline interpolation for 3D human body model surface curvature

\section{Conclusion}

Analysis of the constraint relation in 3D clothing can become the basis of late constraint relationship, based on which the construction of the geometric constraint graph can present specifically the various geometric elements in 3D clothing and their relations. Whether for the clothing CAD or for the construction of 3D human body model, the important role the theory of human body characteristics plays is actually obvious. All in all, this paper makes a brief analysis and description of the problems of clothing CAD parametric technology based on the theory of human characteristics, hoping to provide reference and help for the development of future related researches and practice.

\section{References}

[1] Xia Ping, Yao Jin: The Establishment of Parametric 3D Mannequin Simulation Model Based on Cubic Spline Interpolation , Journal of Chengdu Textile College.2006.23. (04).19-23

[2] Liu Hao: Implementation of Wireless Data Acquisition System of Human Body Parameters in Electronic Intelligent Clothing, Journal of Beijing Institute of Clothing Technology (Natural Science Edition). 2011.31. (01).43-49.59

[3] Qin Ke, Zhuang Yueting, Wu Fei et al: Study on the Parametric 3D Human Model in Clothing CAD, Journal of Computer Aided Design and Computer Graphics.2004.16. (07).918-922

[4] Chen Long, Lu Guodong, Deng Weiyan et al: Study on the Technology of Collision Detection in Parametric Clothing Design , Journal of Image and Graphics.2008.13. (01).162-169

[5] Guo Ruiliang, Zhang Hui: Discussion on Fitting of Plate Curves in Clothing CAD System, Journal of Beijing Institute of Clothing Technology (Natural Science Edition). 2002.22. (02).64-67

[6] Tang Xiaoman, Jiang Lei: The Method of Determination for Children's Size Difference Based on Non-parametric Kernel Density Estimation. , Journal of Beijing Institute of Clothing Technology (Natural Science Edition).2005.25. (04).30-34

[7] Li Jituo, Lu Guodong, Zhang Dongliang et al: Generation Method of Personalized Clothing Based on Sketch Interaction, Journal of Computer Aided Design and Computer Graphics.2005.17. (11).2512-2517

[8] Lee Xiangshuo, Qi Guoning, Yang Qinghai et al: Study on the Modeling Technology of Clothing Model Based on TLAC, 2007.13. Computer Integrated Manufacturing System. (05).856-861 